\title{
Fastidious distributivity*
}

\author{
Jakub Dotlačil \\ University of California Santa Cruz.
}

\begin{abstract}
I argue that nominal predicates cannot be interpreted distributively if their subject is not a distributive quantifier. This is surprising and problematic for the current theories of distributivity. I offer a novel analysis of distributivity, fastidious distributivity, in which only arguments can be interpreted distributively, and formalize fastidious distributivity using team logic.
\end{abstract}

Keywords: distributivity, nominal predicates, team logic

\section{Introduction}

Let me start with basic assumptions about the interpretation of singular and plural entities. I assume that their domain, $D_{e}$, is the power set of the set of all entities (Schwarzschild 1996 among many others). Atomic individuals are singleton sets, non-atomic individuals are unions of atomic individuals. The conjunction of proper names in (1) is the set $\{$ ABELARD, HELOISE $\}$.

(1) Abelard and Heloise are tall.

Example (1) is true iff Abelard is tall and Heloise is tall. These truth conditions are not captured if we assume that tall denotes the function in (2a) since in that case being tall would only be true of the two-membered set, see (2b).

a. $[[$ tall $]]=\lambda x_{e} \cdot \operatorname{TALL}(x)$

b. $[[$ Abelard and Heloise are tall $]]=\operatorname{TALL}(\{$ ABELARD, HELOISE $\})$

To derive the correct truth conditions it is standard to add the $*$-operator (a.k.a the pluralization operator) to semantics, defined (syncategorematically) below.

*-operator (pluralization operator)

a. $[[* P]]=\lambda x . x=x_{1} \cup \ldots \cup x_{n}$ and $P\left(x_{1}\right), \ldots P\left(x_{n}\right)$

* I thank Pranav Anand, Janneke ter Beek, Rajesh Bhatt, Adrian Brasoveanu, Donka Farkas, Jack Hoeksema, Fred Landman, Rick Nouwen, Hotze Rullmann, Anna Szabolcsi, Radek Šimík and Eytan Zweig for discussion and comments. This research was supported by a grant from the Netherlands Organisation for Scientific Research, which I hereby gratefully acknowledge.

(C)2011 Jakub Dotlačil 
b. $[[* R]]=\lambda x y . x=x_{1} \cup \ldots \cup x_{n}$ and $y=y_{1} \cup y_{2} \ldots \cup y_{n}$ and $x_{1} R y_{1}, \ldots x_{n} R y_{n}$

A predicate of any arity can be pluralized but for the purpose of this paper, only pluralized unary and binary predicates are relevant, see (3a) and (3b). In words, a unary predicate that is pluralized is true for an entity if we can split the entity into parts and the predicate holds for the parts, (3a). This split can be trivial. A relation that is pluralized is true for a pair of entities if we can split the entities into parts and pair up the parts in such a way that the relation is true for all such pairs.

Following Krifka (1989), Landman (2000), Kratzer (2008), among many others, I assume that every lexical expression is born plural. Thus, tall should be interpreted as in (4). Furthermore, the non-pluralized function of tall has lexical restrictions: its domain consists only of atomic individuals. Given these two assumptions, (1) is derived to be true iff Abelard is tall and Heloise is tall.

$$
[[\text { tall }]]=* \lambda x . \operatorname{TALL}(x)
$$

It turns out that the $*$-operator on lexical expressions is not sufficient when we analyze interpretations of plural expressions. Consider (5), which is true if each of several women wore a different blue dress. This is the distributive reading of (5).

(5) Several women wore a blue dress.

So far we cannot capture this reading. The problem is that even if we assume that all the lexical expressions in (5) are pluralized, the interpretation we get says that there was one dress and each of the several women wore it, (6).

$$
\begin{aligned}
& [\text { [several women wore a blue dress }]]= \\
& \exists x[|x|=\operatorname{SEVERAL} \wedge * \operatorname{WOMAN}(x) \wedge \exists y[* \operatorname{B} . \operatorname{DRESS}(y) \wedge|y|=1 \wedge * \operatorname{WORE}(x, y)]]
\end{aligned}
$$

This problem can be avoided if we assume that the $*$-operator can also apply to predicates created in syntax. Such $*$-operator is commonly called the distributivity operator. I will follow the tradition and call it that and abbreviate it as $D$ in examples. It is sometimes (but not always) assumed that the predicate modified by the distributivity operator must hold for singletons. This assumption is not crucial for the paper but it is going to simplify the discussion throughout, so I will follow it. Thus, the distributivity operator is defined as in (7a). When the distributivity operator applies to the predicate wore a blue dress, we derive correctly that women (may) wear different dresses, see (7b).

a. $\left[[D(P)]=\lambda x \cdot x=x_{1} \cup \ldots \cup x_{n}\right.$ and $\left|x_{1}\right|=1, \ldots\left|x_{n}\right|=1$ and $P\left(x_{1}\right), \ldots P\left(x_{n}\right)$

b. $[$ several women $D$ (wore a blue dress) $]]=$ $\exists x[|x|=\operatorname{SEVERAL} \wedge * \operatorname{WOMAN}(x) \wedge D$ (wore a blue dress $)]=$ $\exists x\left[|x|=\operatorname{SEVERAL} \wedge * \operatorname{WOMAN}(x) \wedge \forall x^{\prime} \subseteq x\left[\left|x^{\prime}\right|=1 \rightarrow x^{\prime}\right.\right.$ wore a b. dress $\left.]\right]$ 
Aside from distributive quantifiers like each boy or every boy, all DPs that give rise to a distributive interpretation only optionally do so. For example, (8) can be interpreted distributively or collectively, i.e., each boy might have built his own raft (the distributive reading) or the boys might have collaborated in building one raft (the collective reading). I will call the class of DPs that can give rise to distributive and non-distributive interpretations optionally distributive DPs.

(8) Several boys/three boys/all the boys built a raft.

To model the distributive interpretation of an optionally distributive DP one commonly assumes that the distributivity operator can be optionally inserted in syntax (Massey 1976, Roberts 1990, Link 1998, Winter 2001 and many others) or the DP is ambiguous between its distributive and non-distributive interpretation (Winter 2001 and Zweig 2008 for DPs headed by all and some other quantifiers). Either way, what all accounts share is the assumption that optionally distributive DPs distribute over the predicate that is their syntactic scope. We have just seen this in the example above in which in order to interpret (5) distributively, the distributivity operator had to modify the predicate wore a blue dress, i.e., the scope of the subject.

In this paper I am going to argue that contrary to all accounts of distributivity DPs do not distribute over their syntactic scope.

The argument is rather simple: in some cases we see that distribution over the scope of a DP is impossible. In particular, optionally distributive DPs cannot distribute over nominal predicates. (9a)-(9d) get rather nonsensical interpretations. For example, (9a) could only be true if several women as a whole made one pediatrician.

a. \# Several women I know are a pediatrician.

b. \# My friends are a really good teacher.

c. \# I consider all my students a good listener.

d. \# They seem to me to be a promising writer.

The fact that optionally distributive DPs cannot distribute over nominal predicates is in fact commonly exploited (but unacknowledged) when showing that some predicates are non-homogeneous. The following example showing that $a$ dog is a non-homogeneous predicate and thus does not license the inference from (10a) to (10b) is taken from Rothstein (2001).

(10) a. Spot is a dog and Max is a dog.

b. $\nRightarrow \Rightarrow$ Spot and Max are a dog.

Let me focus on (9a) for a second. For sake of illustration, I will follow Landman's approach to predication. The DP a pediatrician starts out as a predicate and it is raised to a quantifier type only when it is interpreted as an argument (thus, this 
option is irrelevant here). Concretely, a pediatrician is interpreted as in (11a). The predicate combines with the subject, giving rise to (11b). This states that there are several women and they together are one pediatrician. Given our world knowledge that one pediatrician is one human being, we correctly derive that (9a) is awkward.
a. $[[$ a pediatrician $]=\lambda x . * \operatorname{PEDIATRICIAN}(x) \wedge|x|=1$
b. $[[(9 \mathrm{a})]]=\exists x[|x|=\operatorname{SEVERAL} \wedge * \operatorname{WOMAN}(x) \wedge * \operatorname{PEDIATRICIAN}(x) \wedge|x|=1]$

The problem is that we also have the distributivity operator at our disposal. We need to postulate it because of the distributive reading of (5) and similar examples. Unfortunately, its existence backfires here. There is no reason to assume it cannot apply to the predicate a pediatrician, (11a) above, which gives us (12a). This, when combined with the subject, leads to (12b). As one can see, (12b) says that each of several women is a pediatrician: a completely natural interpretation that is however missing from (9a). It should be easy to see that the same problem arises in (9b)-(9d) and (10a) now incorrectly entails (10b). Even though I explicitly discussed only Landman's approach to predication, other approaches lead to the same problem. For instance, assuming that indefinites start as quantifiers and are only then lowered to a predicate (Partee 1987 and many others) does not circumvent the problem since the $D$-operator could apply after a pediatrician has been lowered to a predicate.

$$
\begin{aligned}
& \text { a. } D(\lambda x . * \text { pediatrician }(x) \wedge|x|=1) \\
& \text { b. }\left[[(9 a)]=\begin{array}{rl}
= & \exists x[|x|=\operatorname{SEVERAL} \wedge * \operatorname{WOMAN}(x) \\
& \wedge x \in D(\lambda x . * \operatorname{PEDIATRICIAN}(x) \wedge|x|=1) \\
= & \exists x[|x|=\operatorname{SEVERAL} \wedge * \operatorname{WOMAN}(x) \\
& \left.\wedge \forall x^{\prime} \subseteq x\left[x^{\prime}=1 \rightarrow * \operatorname{PEDIATRICIAN}\left(x^{\prime}\right) \wedge\left|x^{\prime}\right|=1\right]\right]
\end{array}\right.
\end{aligned}
$$

Thus, we arrive at conflicting results. On one hand, we need the distributivity operator because of cases like (5), on the other hand, we do not want it, because of cases like (9a). In the rest of the paper, I am going to offer a solution to this problem. In particular, I will argue that optionally distributive DPs do not distribute over their scope. Rather, they distribute only over arguments embedded in their scope. This straightforwardly explains the lack of distributive readings in (9a)-(9d) and (10b) since nominal predicates are not arguments.

The rest of the paper is organized as follows. In Section 2 I discuss alternative attempts to explain the lack of distributive readings in nominal predication. I focus on two explanations: first, distributive readings might be missing because nominal predicates must agree in number with the subject, as discussed in Section 2.1; alternatively, the $D$-operator might be blocked from insertion in nominal predicates, as discussed in Section 2.2. I argue that both explanations are problematic. In Section 3 I develop my own analysis in which distributivity operators do not modify the scope of optionally distributive DPs, rather, they appear directly on arguments inside 
the scope. I call this analysis of distributivity fastidious distributivity. As we will see, it is most straightforwardly captured in frameworks which model dependencies between variables. In Section 4, I use team logic (Väänänen 2007) to account for fastidious distributivity.

\section{Problematic solutions}

In this section I discuss two potential solutions as to why nominal predicates cannot be interpreted distributively if the subject is an optionally distributive DP. I argue that it is problematic to explain this as a consequence of agreement between a nominal predicate and the subject, Section 2.1, and it will also not do to stipulate that the $D$-operator cannot be inserted in nominal predicates, Section 2.2.

\subsection{Problematic solution \#1: Nominal predicates agree with subjects}

de Swart, Winter \& Zwarts (2007) notice that in Dutch (13a) is good, unlike (13b).

(13) a. Jan en Sofie zijn leraren.

Jan and Sofie are teachers

b. \# Jan en Sofie zijn een leraar.

Jan and Sofie are a teacher

Their solution to the unacceptability of $(13 b)$ is that "the marked nominals een leraar in [(13b)] and leraren in [(13a)] have to agree in number with the subject." Thus, the distributive reading of (13b) is excluded on syntactic grounds.

I have reservations about this explanation. First, the agreement between a nominal predicate and the subject would be of a very special type. It would be agreement in number but not other categories, as can be shown in morphologically more rich languages. In Czech nouns are bearers of number, gender and case categories and words agreeing with nouns must match these categories if they can express them. However, nominal predicates do not need to match gender or case of the subject. A mismatch in gender is shown in (14).

$$
\begin{aligned}
& \text { On byl bab-a. (Czech) } \\
& \text { He.sg.masc was.masc hag-sg.fem } \\
& \text { 'He was a coward.' }
\end{aligned}
$$

Thus, the agreement between a nominal predicate and the subject would target number and have to ignore other categories. Since it is unlike any other instances of agreement in Czech in this respect, it is questionable whether any agreement between a nominal predicate and the subject actually takes place. 
Second, the agreement between a nominal predicate and the subject would have to be sensitive to the distinction between collective and distributive readings in English. It would have to be obligatory in case of a distributive reading but optional (or blocked) in case of a collective reading. This is because collectively interpreted nouns do not need to match the number of the subject, see (15), where the first example is taken from the Corpus of Contemporary American English.

(15) a. They survive because they understand they are a team. (COCA)

b. Those five stars are a constellation. (Schwarzschild 1996: p. 179)

This is surprising since agreement in English is not sensitive to the distinction between collective and distributive interpretations. One can see this in (15), where the copula is in plural even though the predicates are interpreted collectively.

A parallel argument can be built regarding pluralia tantum. ${ }^{1}$ Consider the contrast between (16a) and (16b). On the agreement story (16a) should be ungrammatical due to mismatch in number and (16b) should be true if we talk about one or more pairs of scissors. Both predictions are incorrect.

a. These scissors are a very handy tool. (= one object)

b. These scissors are very handy tools. (= more than one object)

Finally, going back to Czech we can see the reverse case of number mismatch, i.e., in which the subject is in singular and a nominal predicate is in plural. First, as a background, notice that some vague numerals are syntactically singular (see Veselovská 2001). Hromada 'loads of' is one such expression, (17a). If this vague numeral is the subject, the corresponding nominal predicate must appear in plural (unless it is interpreted collectively). Hence, (17b) is awkward and (17c) fine.

a. Hromad-a kluků spal-a v predsíni. pile-fem boys slept-3rd.sg.fem in corridor 'Loads of boys slept in the corridor.'

b. \# Hromada kluků byl-a dobr-ým posluchač-em. pile boys was-fem good-sg listener-sg 'Loads of boys were a good listener.'

c. Hromada kluků byl-a dobr-ými posluchač-i. pile boys was-fem good-pl listener-pl 'Loads of boys were good listeners.'

This is not what one would predict if there was agreement in number between a nominal predicate and the subject. In that case, (17b) should be good and (17c) ungrammatical.

1 I am thankful to Rajesh Bhatt for this observation. 
Fastidious distributivity

It is worth noting that all the data problematic for agreement would be accounted for if we block a distributive reading of nominal predicates when the subject is an optionally distributive DP. Singular collective nouns should be able to appear as nominal predicates since no distributivity operator is needed in that case, (15). Singular nominal predicates could predicate over pluralia tantum if the subject denoted one entity, (16a). Finally, the Czech data in (17b) and (17c) would be straightforward, (17b) being bad because the nominal predicates lack distributive readings and $(17 \mathrm{c})$ being acceptable since no distributivity needs to take place here.

Thus, I conclude that nominal predicates lack a distributive reading. However, standard accounts of distributivity cannot capture this restriction, as I show in the next section.

\subsection{Problematic solution \#2: D cannot apply to nominal predicates}

Consider (18a). This sentence can have a distributive reading, namely, each of several (all) women seemed to a different doctor to be crazy. In standard accounts of distributivity this would be taken to show that the subject distributes over the rest of the clause. Using the $D$-operator, the structure to produce the reading must be as shown in (18b).

(18) a. Several women/all the women seemed to a doctor to be crazy.

b. Several women/all the women $D$ (seemed to a doctor to be crazy).

Thus, (18a) gives us independent evidence that the matrix subject can distribute over its predicate. However, (19a) leads to the exactly opposite conclusion. This sentence is awkward, obviously because the subject cannot distribute over the nominal predicate a dangerous patient.

a. \# Several women/all the women seemed to a doctor to be a dangerous patient.

b. Several women/all the women $D$ (seemed to a doctor to be a dangerous patient).

But how could that be? If we state that $D$ cannot modify a seem-clause in (19a) we incorrectly exclude the distributive reading of (18a) as well. We could state something like "an argument cannot distribute over a clause if there is a nominal predicate embedded inside the clause that should apply to the argument". However, I do not see how such a statement could be captured in syntax. ${ }^{2}$

I thus follow a different route: rather than using the standard account of distributivity and blocking its application in special cases, I develop a new treatment of

2 Eytan Zweig pointed out to me that in contrast with (19a) the following sentence is good:

(1) Several women wanted to be a doctor. 
distributivity from which all the data discussed so far follow. Such an analysis of distributivity is the topic of the rest of the paper.

\section{Fastidious distributivity}

When dealing with distributivity semanticists assume that the scope of an optionally distributive DP is interpreted distributively, either due to the meaning of the DP or due to the insertion of the $D$-operator. Then, any arguments embedded inside the scope of the DP can show variation or not. Concretely, consider (20a). To let a single argument vary with respect to the subject some women, the whole predicate must be modified by the distributivity operator, (20b).

(20) a. Some women gave a flower to three men because a shop was nearby.

b. Some women $\mathrm{D}$ (gave a flower to three men because a shop was nearby).

I call this way of dealing with distributivity indiscriminating distributivity. Such a distributivity operator sits on top of the whole scope of a DP and cannot discriminate which arguments inside it might show variation, hence the name.

As an alternative, I am proposing fastidious distributivity. In this analysis, an operator that triggers a distributive reading modifies arguments directly. To distinguish it from the standard distributivity operator, I notate it as $\delta .{ }^{3}$ Concretely, if all arguments in (20a) should vary with respect to the matrix subject, each of them would have to be modified by $\delta$ bound by the subject, (21). The $\delta$-operator obviously discriminates which arguments might show variation, hence the name.

(21) Some women gave $_{x}$ (a flower) to $\delta_{x}$ (three men) because $\delta_{x}$ (a shop) was nearby.

This way of looking at distributivity is not novel. Williams (1991) argued for the same treatment of distributivity based on data from reciprocal sentences. So did Choe (1987) based on Korean "anti-quantifiers". However, these works did not offer a compositional account of fastidious distributivity. Fastidious distributivity is closely related to distributivity over thematic roles in event semantics. I'll say more about this in the next section. It is also closely related to the analysis of scope of indefinites as evaluation dependence of variables, as in Donka Farkas' work,

This is expected since want relates the subject and the embedded attitude. As other verbs, it is pluralized, so we get $*$ WANT(several women,be a doctor). Due to the $*$-operator, this is true if each of several women wanted to be a doctor. This option is not available in (19a) because the matrix subject is not an argument of seem. I am thankful to Pranav Anand for a detailed discussion of this issue.

3 Using this notation anticipates the actual interpretation. The distributivity operator implementing fastidious distributivity is going to be interpreted in a similar way as the distributivity operator, notated as $\delta$, in van den Berg (1996), Nouwen (2007) and Brasoveanu (2010). 
see Brasoveanu \& Farkas (2009). However, fastidious distributivity is restricted to non-distributive quantifiers, which makes it different from the analysis of scope of indefinites in Brasoveanu \& Farkas (2009).

Before moving to the analysis, I would like to point out that there is one easy way to compare indiscriminating and fastidious distributivity. While indiscriminating distributivity assumes that the covert trigger of distributivity is like floating each, in fastidious distributivity the covert trigger of distributivity might be thought of as binominal each. This is similar to the idea of Beghelli \& Stowell (1997) to relate distributivity to each rather than every and to Link's claim that the overt counterpart of the $D$-operator is je in German, which forces a distributive reading. The problem with relating $j e$ to distributivity is that je, like binominal each appears inside a noun phrase (see Zimmermann 2002). This led Link to conclude (Link 1998: 131) that the $\mathrm{D}$-operator and $j e$ are equivalent but je violates surface compositionality. Fastidious distributivity treats the covert distributivity trigger and its overt counterpart, binominal each and the German je, as syntactically identical and no surface compositionality needs to be violated.

\section{Analysis of fastidious distributivity}

The distributive reading of (22a), each of several women read a different book, should be captured by the LF structure (22b). I assume that the $\delta$-operator can apply only to the DPs that are in argument positions. Here, $\delta$ applies to the predicate $a$ book, and the DP is then lifted to a quantifier to play a role of an argument.

a. Several women read a book.

b. Several women ${ }_{x}$ read $\delta_{x}$ (a book).

What could be the meaning of $\delta$ ? The first attempt one might try is something like (23). But obviously, this is not going to work.

$$
\left[\left[\delta_{x}(\mathrm{P})\right]\right]=\lambda y \cdot \forall x^{\prime} \subseteq x\left[\left|x^{\prime}\right|=1 \rightarrow P(y)\right] \text { (first attempt) }
$$

The problem is that the universal quantification binds nothing in the consequent and thus, its application is vacuous. That it is vacuous should be clear from the derivation below. First, (24a) shows the meaning of the distributively interpreted predicate. (24b) shows the meaning of the whole sentence. As should be clear from the last line, we only get the interpretation "there was one book and all women read it". This is not what we want.

$$
\begin{aligned}
& \text { a. }\left[\left[\delta_{x}(\mathrm{a} \text { book })\right]=\lambda y \cdot \forall x^{\prime} \subseteq x\left[\left|x^{\prime}\right|=1 \rightarrow[* \operatorname{BOOK}(y) \wedge|y|=1]\right]\right. \\
& \text { b. }[[(22 \mathrm{~b})]= \\
& \quad \exists x y[|x|=\operatorname{SEVERAL} \wedge * \operatorname{WOMAN}(x) \wedge * \operatorname{READ}(x, y) \\
& \left.\quad \wedge \forall x^{\prime} \subseteq x\left[\left|x^{\prime}\right|=1 \rightarrow[* \operatorname{BOOK}(y) \wedge|y|=1]\right]\right]
\end{aligned}
$$




$$
\begin{gathered}
\Leftrightarrow(\text { Simple first-order equivalences) } \\
\exists x y[|x|=\operatorname{SEVERAL} \wedge * \operatorname{WOMAN}(x) \wedge * \operatorname{READ}(x, y) \\
\left.\wedge\left[\neg \exists x^{\prime}\left[x^{\prime} \subseteq x \wedge\left|x^{\prime}\right|=1\right] \vee[* \operatorname{BOOK}(y) \wedge|y|=1]\right]\right] \\
\Leftrightarrow(\text { Distributive law }) \\
\exists x y\left[\left[|x|=\operatorname{SEV} . \wedge * \operatorname{WOMAN}(x) \wedge * \operatorname{READ}(x, y) \wedge \neg \exists x^{\prime}\left[x^{\prime} \subseteq x \wedge\left|x^{\prime}\right|=1\right]\right]\right. \\
\vee[|x|=\operatorname{SEVERAL} \wedge * \operatorname{WOMAN}(x) \wedge * \operatorname{READ}(x, y) \wedge * \operatorname{BOOK}(y) \wedge|y|=1]] \\
\Leftrightarrow(\text { Quantized expressions }(\operatorname{sev} . \text { women }) \text { have atoms, Krifka 1989) } \\
\exists x y[|x|=\operatorname{SEVERAL} \wedge * \operatorname{WOMAN}(x) \wedge * \operatorname{READ}(x, y) \wedge * \operatorname{BOOK}(y) \wedge|y|=1]
\end{gathered}
$$

Using event semantics works better here since one might assume that $\delta$ binds arguments in thematic roles. In fact, event semantics implemented fastidious distributivity on top of the standard indiscriminating distributivity (see Landman 2000; Dotlačil 2010, both following Schein 1993). However, event semantics needs to stipulate more than just a $\delta$-operator. A compositional analysis in event semantics also needs to add extra mechanisms to manipulate thematic roles to make them available to $\delta$ (see, for example, Landman 2000: §10).

I will not follow this line of research since implementing fastidious distributivity is much more straightforward in frameworks studying dependency, which appeared in linguistics, especially dynamic semantics (Krifka 1996, van den Berg 1996, Nouwen 2003, Brasoveanu 2007) and logic (independence-friendly logic, Hintikka 1996, its notational variant dependence logic, Väänänen 2007, and its extension team logic, Väänänen 2007). These frameworks operate with indiscriminating distributivity but they are suitable for fastidious distributivity, as I'll show in the rest of the paper using team logic.

\subsection{A short introduction to team logic}

\subsubsection{Syntax and interpretation of atomic formulas in team logic}

Team logic is a recently developed logic (Väänänen 2007) which, like dependence logic and independence-friendly logic, is useful for studying dependencies between variables. It is closely related to dependence logic in the sense that one arrives at team logic by adding classical negation to dependence logic (dependence logic has its own negation which does not satisfy the law of excluded middle). Team logic is equivalent in its expressive power to full second-order logic (Väänänen 2007, Kontinen \& Nurmi 2009).

The syntax of team logic is almost identical to first-order logic, the only difference being that team logic adds new connectives and a new atomic formula and has two negations. Not all these additions are relevant for the purpose of this paper, in particular, the role of non-classical negation is not, so I ignore it here. The formulas 
of atomic form are summarized in the table in (25a), where $t$ with subscripts are terms, i.e., individual constants or variables. The formulas of compound form are summarized in the table in $(25 \mathrm{~b})$, where Greek letters are themselves formulas.

a.

\begin{tabular}{ll}
\hline \hline Atomic formula & Name \\
\hline$t_{1}=t_{n}$ & equivalence \\
$t_{1} \neq t_{n}$ & difference \\
$R\left(t_{1}, \ldots t_{n}\right)$ & relation \\
$=\left(t_{1}, \ldots, t_{n-1}, t_{n}\right)$ & dependence \\
\hline
\end{tabular}

\begin{tabular}{ll}
\hline \hline Compound & Name \\
\hline$\phi \wedge \psi$ & conjunction
\end{tabular}

b.

\begin{tabular}{ll}
$\phi \vee \psi$ & disjunction \\
$\phi \otimes \psi$ & tensor \\
$\sim \phi$ & negation (classical) \\
$\exists x \phi$ & existential \\
$\forall x \phi$ & universal \\
\hline
\end{tabular}

Traditionally, independence-friendly logic, on which team logic is built, had been interpreted in game-theoretic terms as a game of two players with imperfect information (Hintikka 1996). There is also an alternative interpretation using sets of assignment functions (Hodges 1997, Väänänen 2007), which I will follow here.

In first-order logic formulas are satisfied relative to single assignments. In dependence logic and team logic we move one level up and consider sets of assignments. This allows us to model dependencies between variables. Unlike the game-theoretic interpretation, the interpretation using sets of assignments is straightforwardly compositional. Väänänen (2007) calls sets of assignments teams, hence the name of this logical framework.

Given model $\mathscr{M}$ consisting of the domain $M$ and interpretation function $I$, and a set of assignments $G$, we define the semantics of atomic formulas as in (26). A term is interpreted in the same way as in first-order logic, that is, as the value of the interpretation function of $\mathscr{M}$ if it is a constant and as the value of $g, g \in G$ if it is a variable. Of the interpretations of atomic formulas, (26d) is probably the least transparent. I will first focus on (26a)-(26c) and come back to (26d) in a minute.

(26) Interpretation of atomic formulas:
a. $\left[\left[t_{1}=t_{n}\right]\right]^{\mathscr{M}, G}=\mathrm{T}$ if and only if (=iff) for each $g \in G,\left[\left[t_{1}\right]\right]^{\mathscr{M}, g}=\left[\left[t_{n}\right]\right]^{\mathscr{M}, g}$
b. $\left[\left[t_{1} \neq t_{n}\right]\right]^{\mathscr{M}, G}=\mathrm{T}$ iff for each $g \in G,\left[\left[t_{1}\right]\right]^{\mathscr{M}, g} \neq\left[\left[t_{n}\right]\right]^{\mathscr{M}, g}$
c. $\left[\left[R\left(t_{1}, \ldots t_{n}\right)\right]\right]^{\mathscr{M}, G}=\mathrm{T}$ iff for each $g \in G,\left\langle\left[\left[t_{1}\right]\right]^{\mathscr{M}, g}, \ldots,\left[\left[t_{n}\right]\right]^{\mathscr{M}, g}\right\rangle \in I(R)$ 
d. $\left[\left[=\left(t_{1}, \ldots, t_{n}\right)\right]\right]^{\mathscr{M}, G}=\mathrm{T} \quad$ iff for each $g, g^{\prime} \in G$, such that

$$
\begin{aligned}
& {\left[\left[t_{1}\right]\right]^{\mathscr{M}, g}=\left[\left[t_{1}\right]\right]^{\mathscr{M}, g^{\prime}}, \ldots\left[\left[t_{n-1}\right]\right]^{\mathscr{M}, g}=\left[\left[t_{n-1}\right]\right]^{\mathscr{M}, g^{\prime}}} \\
& \text { it holds that }\left[\left[t_{n}\right]\right]^{\mathscr{M}, g}=\left[\left[t_{n}\right]\right]^{\mathscr{M}, g^{\prime}}
\end{aligned}
$$

As shown in (26a)-(26c), relations, differences and equivalences are checked distributively, that is, in each assignment function. To see how this works, consider the following set $G$, consisting of three assignment functions: $g_{1}, g_{2}$ and $g_{3} . g_{1}$ assigns Heloise to $x$ and Arnold to $y . g_{2}$ assigns Abelard to $x$ and Arnold to $y$. $g_{3}$ assigns Arnold to $x$ and Arnold to $y{ }^{4}$ As is standard, we summarize a set of assignment functions by using a matrix. In the matrix each row represents values of one assignment function and each column represents values of one variable in all assignment functions. The matrix in (27) shows the summary of $G$ for $x$ and $y$.

\begin{tabular}{ccc} 
& $x$ & $y$ \\
\hline \hline$g_{1}$ & $\{$ HeloisE $\}$ & $\{$ ABElaRD $\}$ \\
$g_{2}$ & $\{$ ABELARD $\}$ & $\{$ ARNOLD \\
$g_{3}$ & $\{$ ARNOLD & $\{$ ArNOLD \\
\hline
\end{tabular}

In this particular set and assuming that Heloise likes Abelard, Abelard likes Arnold and Arnold likes himself, (28a) is true (because Abelard is a man and Arnold is a man), (28b) is not true and (28c) is true. Furthermore, (28d) is not true (because of the values of $x$ and $y$ in $g_{1}$ and $g_{2}$ ) and neither is (28e) (because of the values of $x$ and $y$ in $\left.g_{3}\right)$ !
a. $\operatorname{MAN}(y)$
b. $\operatorname{WOMAN}(x)$
c. $\operatorname{LIKE}(x, y)$
d. $x=y$
e. $x \neq y$

The last atomic formula that we have put aside so far, interpreted in (26d), expresses dependence between variables. Its intuitive meaning is that the value of the term $t_{n}$ depends only on the values of the terms $t_{1}, \ldots t_{n-1}$. Put differently, one could in principle define a function that would compute the value of $t_{n}$ from the values of $t_{1} \ldots t_{n-1}$. In our example, (29a) is true but (29b) is not. (29b) is not true because in $g_{2}$ and $g_{3}, y$ stays the same but $x$ varies.
a. $=(x, y)$
b. $=(y, x)$

4 The values of the variables are singleton sets. Recall the discussion in Section 1 that both atomic and non-atomic entities are treated as sets. 
Dependence is a useful tool to model variation of indefinites with respect to a distributive quantifier (see Brasoveanu \& Farkas 2009). I will use dependence to capture cardinality conditions.

\subsubsection{Interpretation of compounds and cardinality conditions}

In (30) below, I summarize the interpretation of unary and binary connectives. The definitions of conjunction and negation are basically the same as in first-order logic. So is disjunction, which in fact is not a primitive but can be derived from conjunction and negation. Tensor, defined in (30c), is interpreted as a split of the set of assignment functions. The formulas $\phi$ and $\psi$ conjoined by tensor need to hold only in one subset of $G$ each.

(30) Interpretation of unary and binary connectives:

a. $[[\phi \wedge \psi]]^{\mathscr{M}, G}=\mathrm{T}$ iff $[[\phi]]^{\mathscr{M}, G}=\mathrm{T}$ and $[[\psi]]^{\mathscr{M}, G}=\mathrm{T}$

b. $[[\phi \vee \psi]]^{\mathscr{M}, G}=\mathrm{T}$ iff $[[\phi]]^{\mathscr{M}, G}=\mathrm{T}$ or $[[\psi]]^{\mathscr{M}, G}=\mathrm{T}$

c. $\quad[\phi \otimes \psi]]^{\mathscr{M}, G}=\mathrm{T} \quad$ iff $G=G_{1} \cup G_{2}$ and $G_{1} \neq \emptyset, G_{2} \neq \emptyset$ and $[[\phi]]^{\mathscr{M}, G_{1}}=\mathrm{T}$ and $[[\psi]]^{\mathscr{M}, G_{2}}=\mathrm{T}$

d. $[[\sim \phi]]^{\mathscr{M}, G}=\mathrm{T}$ iff $[[\phi]]^{\mathscr{M}, G} \neq \mathrm{T}$

As an example of the work of tensor, notice that in the set of assignments discussed above, (27), neither (31a) nor (31b) is true because there are men and women as values of $x$. However, (31c) is true because we can split $G$ into two subsets, $\left\{g_{1}\right\}$, which satisfies the first conjunct and $\left\{g_{2}, g_{3}\right\}$, which satisfy the second conjunct.
a. $\operatorname{WOMAN}(x)$
b. $\operatorname{MAN}(x)$
c. $\operatorname{WOMAN}(x) \otimes \operatorname{MAN}(x)$

Tensor plays an important role in defining the $\delta$-operator, as we will see below. It is also relevant for defining cardinality conditions. Brasoveanu (2008) argues that cardinality should be interpreted collectively relative to assignments. In other words, cardinality conditions should not operate on individual assignments, as other conditions, rather, they should operate on assignments put together. This is also crucial for the analysis of cumulative readings, see Brasoveanu (2010). This means that we should interpret cardinality conditions as follows:

(32) The desired interpretation of cardinality conditions:

$$
[[|t|=n]]^{\mathscr{M}, G}=\mathrm{T} \text { iff }\left|\bigcup\left\{[[t]]^{\mathscr{M}, g}: g \in G\right\}\right|=n
$$

Unfortunately, cardinality conditions are not interpreted this way in team logic. In fact, they are interpreted distributively, that is, in the same way as any other 
conditions. I notate the actual interpretation of cardinality conditions in team logic as $n(t)$, which should be read as $t$ is of cardinality $n$ in each assignment $g \in G$.

(33) The actual interpretation of cardinality conditions in team logic:

$$
[[n(t)]]^{\mathscr{M}, G}=\mathrm{T} \text { iff for each } g \in G,\left|[[t]]^{\mathscr{M}, g}\right|=n
$$

Thus, (32) would add a new atomic formula. Fortunately, we can think of (32) simply as an abbreviation of a complex team logic formula using tensors and dependence. First, notice that there is one extreme case of dependence, namely:

$$
=(t)
$$

This says that $t$ is dependent on no other term, that is, it is independent. In other words, regardless of any variable, $t$ stays the same. Crucially, if $t$ is an atom then (34) says that $t$ has cardinality one in all assignments put together. Cardinality two can then be defined as:

$$
|t|=2:=[=(t) \wedge 2(t)] \vee[\sim=(t) \wedge[[=(t) \wedge 1(t)] \otimes[=(t) \wedge 1(t)]]]
$$

According to the formula in (35) there are two ways that $t$ can have cardinality 2 . First, given the first disjunct, there is only one $t$ for all assignments which is of cardinality two. Alternatively, if we split the set of assignments $G$ into two subsets, $t$ has one value in each subset, that is, it is of cardinality two in total. Either way, $t$ will have the total cardinality 2 . We can generalize to any cardinality $n$ of a term in this way, as shown below, where $\otimes^{n} \phi$ means that $\phi$ is repeated $n$ times and the formulas are conjoined by tensor.

$$
|t|=n:=[=(t) \wedge n(t)] \vee\left[\sim\left[\otimes^{n-1}=(t)\right] \wedge\left[\otimes^{n}[=(t) \wedge 1(t)]\right]\right]
$$

Finally, the last thing we need to consider before we turn to distributivity is the interpretation of quantifiers. In first-order logic, quantification is captured as extension of the assignment function. Recall that in first-order logic $g(a / x)$ denotes a new assignment, $g^{\prime}$, which is like $g$ except that it potentially differs at $x$ to which it assigns the value $a$. In team logic, we generalize extension to sets of assignments:

If $M$ is the domain of a model, $G$ is a set of assignment functions with $M$ as its codomain and $F: G \rightarrow M$, we let $G(F / x)$ denote a new set of assignment functions $\{g(F(g) / x): g \in G\}$

Thus, $G^{\prime}, G^{\prime}=G(F / x)$ is like $G$ except that it potentially differs at $x$ for each $g^{\prime} \in G^{\prime}$ from any $g \in G$.

With this background, we can now interpret existential and universal quantification as shown below. Even though I explicitly mention the interpretation of universal quantification, it is not a primitive. It can be derived from the combination of the existential quantifier and negation in the same way as in first-order logic. 
Interpretation of quantification:

a. $[[\exists x \phi]]^{\mathscr{M}, G}=\mathrm{T}$ iff $[[\phi]]^{\mathscr{M}, G(F / x)}=\mathrm{T}$ for some $F: G \rightarrow M$

b. $[[\forall x \phi]]^{\mathscr{M}, G}=\mathrm{T}$ iff $[[\phi]]^{\mathscr{M}, G(F / x)}=\mathrm{T}$ for every $F: G \rightarrow M$

I conclude this short introduction to team logic with one example, (39a), which gets the LF structure shown in (39b). In the LF structure EC is existential closure. Bracketing expresses the syntactic structure.

a. Several women read a book.

b. EC(several women)(EC(a book) (read))

Several women and $a$ book are translated as formulas with free variables, where the free variables are bound by existential closure, EC. ${ }^{5}$ Read is a relation whose free variables are bound by corresponding existential quantifiers.

$$
\begin{array}{ll}
\text { several women } & \rightsquigarrow|x|=\operatorname{SEVERAL} \wedge \text { WOMAN }(x) \\
\begin{array}{l}
\text { a book } \\
\text { read }
\end{array} & \rightsquigarrow \operatorname{BOOK}(x) \wedge|x|=1 \\
\operatorname{EC}(\phi)(\psi) & \rightsquigarrow \exists x^{\prime}\left[\phi\left[x / x^{\prime}\right] \wedge \psi\left[x / x^{\prime}\right]\right]
\end{array}
$$

Thus, (39b) is interpreted as:

$$
\exists x[|x|=\operatorname{SEVERAL} \wedge \operatorname{WOMAN}(x) \wedge \exists y[\operatorname{BOOK}(y) \wedge|y|=1 \wedge \operatorname{READ}(x, y)]]
$$

This formula is true in the model in which, for example, Heloise, Brenda and Anne each read Freedom, assuming that cardinality three is considered "several". As a starting point to evaluate the formula we might consider the set of assignments $G$ discussed in the examples above. After the introduction of existential quantifiers this set would be extended to a new set, $G^{\prime}$, being:

\begin{tabular}{ccc} 
& $x$ & $y$ \\
\hline \hline$g_{1}^{\prime}$ & $\{$ HELOISE $\}$ & $\{$ FrEEDOM \\
$g_{2}^{\prime}$ & $\{$ BRENDA & $\{$ FrEEDOM \\
$g_{3}^{\prime}$ & $\{$ ANNE & $\{$ FrEEDOM $\}$ \\
\hline
\end{tabular}

This new set obviously makes all conditions in (41) true, that is, the cardinality of the values of $x$ in total is several, the values of the values of $y$ in total is one, and for each assignment $g^{\prime}$ in $G^{\prime}$ it is true that $g^{\prime}(x)$ is a woman, $g^{\prime}(y)$ is a book and $g^{\prime}(x)$ read $g^{\prime}(y)$. Notice that we only derive a non-distributive reading. That is, each of the women must have read the same book. Had they read different books we would end up with more than one value in $y$. In order to derive a distributive interpretation we need to add the $\delta$-operator. I turn to doing so in the next section.

$5 \phi\left[x / x^{\prime}\right]$ denotes the formula which is like $\phi$ but in which free occurences of $x$ are substituted by $x^{\prime}$, assuming $x^{\prime}$ does not occur in $\phi$. 


\section{$4.2 \delta$-operator in team logic}

Consider the LF structure of a distributive reading with $\delta$ correctly taking scope only inside an argument, as we want assuming fastidious distributivity. The subscripts are used to indicate that $\delta$ is bound by the subject.

$$
\mathrm{EC}_{x}(\text { several women })\left(\mathrm{EC}\left(\delta_{x}(\text { a book })\right)(\mathrm{read})\right)
$$

We want to get to the interpretation in which each of the women read one book but the books might be different from each other. This interpretation is achieved if we interpret the scope of $\delta$ only with respect to a subset of assignments. Which subsets? The ones in which the cardinality of $x$ is 1 . This will ensure that the predicate 1 book will be satisfied only with respect to the context "being read by one woman", which is crucial to derive the correct distributive reading.

$$
\text { Distributivity operator } \delta_{x} \text { : }
$$

$$
\left[\left[\delta_{x}[\phi]\right]\right]^{\mathscr{M}, G}=\mathrm{T} \text { iff }[[\phi]]^{\mathscr{M}, G^{\prime}}=\mathrm{T} \text { for each maximal } G^{\prime}, G^{\prime} \subseteq G \text { s.t. }[[|x|=1]]^{\mathscr{M}, G^{\prime}}
$$

We want $\delta$ not to appear freely. I assume that fastidious distributivity is a syntactic requirement on the position of $\delta$.

\section{(45) Syntactic restriction on $\delta: \delta$ can only appear in an argument DP}

The syntactic condition in (45) blocks the distributive interpretation of nominal predicates, i.e., examples like (46a) because in these cases $\delta$ would not apply to an argument, see (46b). Moreover, the interpretation of $\delta$ ensures that nominal predicates will not be interpreted distributively even if higher arguments will, as in \#Several women seemed to a doctor to be a dangerous patient, see Section 2.2.

a. \# Several women are a pediatrician.

b. $\mathrm{EC}_{x}$ (several women) ( are $\delta_{x}($ a pediatrician $)$ )

Ultimately, we want the syntactic restriction on $\delta$ to follow from independent principles but this is something I have to leave for future research.

It is worth noting that the definition of $\delta$, (44), does not add a new primitive to team logic but can be derived from the existing principles. In fact, we can think of $\delta$ as the abbreviation of the team logic formula shown below, where $\phi \rightarrow \psi$ is defined in the same way as in first-order logic, that is, as $\sim \phi \vee \psi$.

$$
\delta_{x} \phi:=\forall z[[|z|=1 \wedge[z=x \wedge \otimes z \neq x]] \rightarrow[[z=x \wedge \phi] \otimes z \neq x]]
$$

Using this definition, we can translate the LF structure of (43) into (48a) which in turn is just an abbreviation of (48b):

$$
\begin{aligned}
& \text { a. } \exists x\left[x=\operatorname{SEVERAL} \wedge \operatorname{WOMAN}(x) \wedge \exists y\left[\operatorname{READ}(x, y) \wedge \delta_{x}[\operatorname{BOOK}(y) \wedge|y|=1]\right]\right] \\
& \text { b. } \exists x[x=\operatorname{SEVERAL} \wedge \operatorname{WOMAN}(x) \wedge \exists y[\operatorname{READ}(x, y) \wedge \\
& \quad \forall z[[|z|=1 \wedge[z=x \otimes z \neq x]] \rightarrow[[z=x \wedge \operatorname{BOOK}(y) \wedge|y|=1] \otimes z \neq x]]]]
\end{aligned}
$$


Let me break down the last formula. The first line introduces several women in position $x$ and something they read in $y$. This leads us to consider an extended set of assignments, $G^{\prime}$, in which we store several women in $x$, and what each read in corresponding values of $y$.

\begin{tabular}{ccc} 
& $x$ & $y$ \\
\hline \hline$g_{1}^{\prime}$ & $\left\{\right.$ WOMAN $\left._{1}\right\}$ & $\left\{\right.$ BOOK READ BY WOMAN $\left._{1}\right\}$ \\
$g_{2}^{\prime}$ & WOMAN $\left._{2}\right\}$ & $\left\{\right.$ BOOK READ BY WOMAN $\left._{2}\right\}$ \\
$\ldots$ & $\ldots$ & $\ldots$ \\
\hline
\end{tabular}

The second line of (48b) makes us consider a new set of assignments, $G^{\prime \prime}$. There are two situations that we should distinguish regarding the values of $z$ in $G^{\prime \prime}$ :

i. All the values of $z$ together have cardinality greater than one or they are of cardinality one but the value is different from any value of $x$. Then, the formula in scope of the universal quantifier in (48b) is true since the antecedent of the conditional is not true.

ii. $z$ has only one value and it is identical to one value of $x$. For example, for each $g^{\prime \prime} \in G^{\prime \prime}, g^{\prime \prime}(z)=\left\{\right.$ WOMAN $\left._{1}\right\}$. Only in that case does the consequent become relevant. We check that the subset of assignment functions in which $z=x$ satisfies the scope of $\delta$. The scope of $\delta$ says that $y$ is one book. Since the value of $y$ is the thing that woman $_{1}$ read we correctly derive that the formula in scope of the universal quantifier is true iff woman $_{1}$ read 1 book (she could have read different books beside this one; this is blocked if pragmatic streghtening applies). Since this procedure is going to be repeated for every possible value $z$ might have we correctly derive that the formula is true iff each of several women read a book and crucially, books might be different from each other.

Thus, (47) correctly derives the distributive reading and together with the syntactic requirement (45) gives us fastidious distributivity. We correctly deal with distributive readings even though $\delta$ has a lower scope than the $D$-operator because we use team logic which passes all relevant information in sets of assignments to $\delta$.

The $\delta$-operator does the same work as the distributivity operator in van den Berg (1996), Brasoveanu (2007), Nouwen (2007), Brasoveanu (2010), among others. However, of these, only Brasoveanu's account is suitable for implementing fastidious distributivity and in particular Plural Compositional DRT (PCDRT) developed in Brasoveanu (2007) should be used if one wants to go fully compositional. Nouwen (2007) and van den Berg (1996) are problematic because they assume that relations are satisfied in the sum of all assignments and because they postulate a different way 
of extending a set of assignment functions. Rather than using $\exists x$, defined above, they operate with the extension $G\left(M^{\prime} / x\right)$, which we can define as follows in team logic:

(50) If $M$ is the domain of a model, $G$ is a set of assignment functions with $M$ as its codomain and $M^{\prime}$ is some subset of $M$, then $G\left(M^{\prime} / x\right)$ denotes a new set of assignment functions $\left\{g(a / x): a \in M^{\prime} \wedge g \in G\right\}$

Readers familiar with dependence and team logic might recognize this definition. $G(M / x)$ is the extension of assignments used in defining the interpretation of the third quantifier of team logic, shriek (notated as $! x$ ). Let's adopt a convention that shriek operating on some subdomain of $M$ is notated as $! x^{M}$. Then, our LF of the distributive reading of (39a) above would be interpreted as follows:

$$
! x^{M}\left[x=\operatorname{SEVERAL} \wedge \text { WOMAN }(x) \wedge ! y^{M}\left[\operatorname{READ}(x, y) \wedge \delta_{x}[\operatorname{BOOK}(y) \wedge|y|=1]\right]\right]
$$

Two shrieks in scope of each other force a branching reading if $\delta$ is missing. In that case, adding $\delta$ in their scope becomes vacuous. On the other hand, two existential quantifiers in scope of each other derive a cumulative reading if $\delta$ is missing. Adding $\delta$ inside an argument then leads to a distributive reading, as we have seen. This leads to the following generalization:

(52) A quantifier $Q$ shows fastidious distributivity only if $Q$ can also participate in a cumulative reading with DPs in its scope

This explains why distributive quantifiers (every, each, including the floating variant of the latter) can distribute over nominal predicates. Since they lack cumulative readings, fastidious distributivity is impossible and they must resort to indiscriminating distributivity. On the other hand, the vast majority of quantifiers that show fastidious distributivity (in other words, cannot distribute over nominal predicates) have cumulative readings. One problematic case might be the quantifier all. However, even this quantifier allows what looks like a cumulative reading, at least with some expressions, like bare plurals (Zweig 2008).

\section{Conclusion}

I have argued that nominal predicates cannot be interpreted distributively when their subject is an optionally distributive DP, which is surprising for the current theories of distributivity. Using team logic, I have offered a novel analysis of distributivity, fastidious distributivity, in which only arguments can be interpreted distributively. Even though I did not offer a compositional account of fastidious distributivity, this could be done if one is willing to switch to PCDRT (Brasoveanu 2007). I leave this extension to another occasion. 
Fastidious distributivity

\section{References}

Beghelli, Filippo \& Timothy Stowell. 1997. Distributivity and negation. In Anna Szabolcsi (ed.), Ways of scope taking, 71-109. Dordrecht: Kluwer.

van den Berg, Martin. 1996. Some aspects of the internal structure of discourse: The dynamics of nominal anaphora. Amsterdam: University of Amsterdam dissertation.

Brasoveanu, Adrian. 2007. Structured nominal and modal reference: Rutgers University dissertation.

Brasoveanu, Adrian. 2008. Donkey pluralities: plural information states versus non-atomic individuals. Linguistics and Philosophy 31(2). 129-209.

Brasoveanu, Adrian. 2010. Modified numerals as post-suppositions. In Maria Aloni, Harald Bastiaanse, Tikitu de Jager \& Katrin Schulz (eds.), Logic, language and meaning, 203-212. Springer.

Brasoveanu, Adrian \& Donka Farkas. 2009. Scope and the grammar of choice. In Proceedings of the tenth symposium of logic and language, Budapest.

Choe, Jae-Woong. 1987. Anti-quantifiers and a theory of distributivity. Amherst: University of Massachusetts dissertation.

Dotlačil, Jakub. 2010. Anaphora and distributivity. A study of same, different, reciprocals and others. Utrecht: Utrecht University dissertation.

Hintikka, Jaako. 1996. Principles of mathematics revisited. Cambridge: Cambridge University Press.

Hodges, Wilfrid. 1997. Compositional semantics for a language of imperfect information. Logic Journal of the IGPL 5. 539-563.

Kontinen, Juha \& Ville Nurmi. 2009. Team logic and second-order logic. In Hiroakira Ono, Makoto Kanazawa \& Ruy de Queiroz (eds.), Logic, language, information and computation, vol. 5514 Lecture Notes in Computer Science, 230-241. Springer Berlin / Heidelberg. http://dx.doi.org/10.1007/ 978-3-642-02261-6_19.

Kratzer, Angelika. 2008. On the plurality of verbs. In Johannes Dölling, Tatjana Heyde-Zybatow \& Martin Schäfer (eds.), Event structures in linguistic form and interpretation, Berlin/New York: Mouton de Gruyter.

Krifka, Manfred. 1989. Nominal reference, temporal constitution, and quantification in event semantics. In Renate Bartsch, Johan van Bentham \& Peter van Emde Boas (eds.), Semantics and contextual expressions, Dordrecht: Foris.

Krifka, Manfred. 1996. Parametrized sum individuals for plural reference and partitive quantification. Linguistics and Philosophy 19. 555-598.

Landman, Fred. 2000. Events and Plurality: The Jerusalem Lectures. Dordrecht: Kluwer.

Landman, Fred. 2004. Indefinites and the type of sets. Oxford: Blackwell. 
Link, Godehard. 1998. Algebraic Semantics in Language and Philosophy. Stanford: CSLI Publications.

Massey, Gerald. 1976. Tom, Dick, and Harry, and all the king's men. American Philosophical Quarterly 13. 89-107.

Nouwen, Rick. 2003. Plural pronominal anaphora in context: dynamic aspects of quantification: UIL-OTS, Utrecht University dissertation.

Nouwen, Rick. 2007. On dependent pronouns and dynamic semantics. Journal of Philosophical Logic 36. 123-154.

Partee, Barbara. 1987. Noun phrase interpretation and type-shifting principles. In Jeroen Groenendijk, Dick de Jong \& Martin Stokhof (eds.), Studies in Discourse Representation Theory and the theory of generalized quantifiers, 115-143. Dordrecht: Foris Publications.

Roberts, Craige. 1990. Modal subordination, anaphora, and distributivity. New York and London: Garland Publishing.

Rothstein, Susan. 2001. Predicates and their subjects. Dordrecht: Kluwer Academic Publishers.

Schein, Barry. 1993. Plurals and events. Cambridge, Massachusetts: MIT Press.

Schwarzschild, Roger. 1996. Pluralities. Dordrecht: Kluwer.

de Swart, Henriëtte, Yoad Winter \& Joost Zwarts. 2007. Bare nominals and reference to capacities. Natural language and linguistic theory 25. 195-222.

Väänänen, Jouko. 2007. Dependence Logic: A new approach to Independence Friendly Logic. Cambridge: Cambridge University Press.

Veselovská, Ludmila. 2001. Agreement patterns of Czech group nouns and quantifiers. In Norbert Corver \& Henk van Riemsdijk (eds.), Semi-lexical categories, 273-320. Berlin: Mouton de Gruyter.

Williams, Edwin. 1991. Reciprocal scope. Linguistic Inquiry 22(1). 159-173.

Winter, Yoad. 2001. Flexibility Principles in Boolean Semantics. Cambridge, Massachusetts: MIT Press.

Zimmermann, Malte. 2002. Boys buying two sausages each: On the syntax and semantics of distance-distributivity. Amsterdam: University of Amsterdam dissertation.

Zweig, Eytan. 2008. Dependent plurals and plural meaning. New York: New York University dissertation.

Jakub Dotlačil

Linguistics, UCSC, Stevenson Faculty Services

1156 High Street

Santa Cruz, CA 95064

j.dotlacil@gmail.com 